\title{
Induction of Systemic Resistance to Albugo candida in Brassica juncea by Pre- or Coinoculation with an Incompatible Isolate
}

\author{
U. S. Singh, K. J. Doughty, N. I. Nashaat, R. N. Bennett, and S. J. Kolte
}

First and fifth authors: Department of Plant Pathology, G.B. Pant University of Agriculture and Technology (GBPUAT), Pantnagar, India; second, third, and fourth authors: IACR-Rothamsted, Harpenden, Herts. AL5 2JQ, United Kingdom. Accepted for publication 3 September 1999.

\begin{abstract}
Singh, U. S., Doughty, K. J., Nashaat, N. I., Bennett, R. N., and Kolte, S. J. 1999. Induction of systemic resistance to Albugo candida in Brassica juncea by pre- or coinoculation with an incompatible isolate. Phytopathology 89:1226-1232.

In an investigation of the interaction between two isolates of Albugo candida that were compatible (CO) and incompatible (IN) on a Brassica juncea accession, the IN isolate induced both local and systemic protection of cotyledons and true leaves against the $\mathrm{CO}$ isolate. The extent of the protection was proportional to the zoosporangia concentration used

were inoculated together but was greater when the interval between the IN and CO isolate inoculations was longer. The IN isolate induced only slight protection when it was inoculated after the $\mathrm{CO}$ isolate. No induced susceptibility to the IN isolate occurred with any treatment. There was some evidence of competition between $\mathrm{CO}$ and IN zoospores for infection sites (stomata). The occurrence of systemic protection and changes detected in phenylalanine ammonia lyase and total soluble peroxidase activities in inoculated cotyledons, particularly after the inducing (IN) inoculation, suggested that host-mediated factors also may be involved in the interaction between the two isolates.
\end{abstract} in the inducing (IN) inoculation. Protection was greatest locally on cotyledons and least on true leaves (the most remote tissue from the point of the inducing inoculation). Protection occurred when the two isolates
Additional keywords: compatibility, incompatibility, induced resistance.
White rust, caused by the biotrophic oomycete pathogen Albugo candida (Pers. ex Hook.) Kunze, is an important disease of Brassica juncea (L.) Czernj. \& Coss. and B. rapa L. The pathogen can infect all aboveground parts of the plant, producing characteristic white blisters (sori). Severe infection culminates in systemic "staghead" infection of the inflorescence (often in association with Peronospora parasitica), which is the main cause of yield loss in susceptible cultivars $(2,3,16)$.

A. candida exhibits specialization on different cruciferous species and cultivars within species (16). The North American population of the pathogen has been categorized into races that cause the most severe disease on their respective homologous hosts, although some also are capable of infecting heterologous hosts $(13,17)$.

In some host-pathogen systems, prior inoculation with an incompatible isolate can protect plants against subsequent infection by a compatible isolate. There has been much interest in this type of induced resistance, because it suggests the possibility of "immunizing" crops against disease $(10,18)$. Little work has been done on the interactions among isolates of pathogens differing in virulence on Brassica hosts, although Mahuku et al. (12) recently demonstrated that highly and weakly virulent isolates of Leptosphaeria maculans can coexist in lesions on B. napus and described how isolates can interact during disease development. The aim of our study was to determine the interaction between incompatible (IN) and compatible (CO) isolates of $A$. candida on B. juncea. We measured isolate interaction in terms of symptom expression and did preliminary investigations of physical (competition for infection sites among zoospores) and host-mediated (defense-related enzymes) factors associated with the interaction.

Corresponding author: N. I. Nashaat; E-mail address: Nash.Nashaat@bbsrc.ac.uk

Publication no. P-1999-1014-01R

C 1999 The American Phytopathological Society

\section{MATERIALS AND METHODS}

Fungal isolates. Two single-pustule isolates of $A$. candida, originally collected by N. I. Nashaat at Pantnagar in northern India during January 1995 (MAFF import license PHF 1307C/1253/114), were used. The incompatible (with $B$. juncea) isolate (IA01A) was collected from toria (B. rapa); the compatible isolate (IA102A) was collected from mustard (B. juncea). The isolates were maintained separately on seedlings of accessions of the hosts from which they were originally collected: PT303 (B. rapa) and Kranti (B. juncea).

Plant material. A B. juncea accession (PPBJ-1) was used as the host throughout the experiments. Seedlings were raised from untreated seed, either in 8 -cm-diameter plastic pots or in $5-\mathrm{cm}^{2}$ cardboard jiffy pots (Nursery Trades [Lee Valley] Ltd., Cheshunt, U.K.). Seeds were sown $\approx 1 \mathrm{~cm}$ deep in a soilless peat-based compost mix (Petersfield Products, Cosby, U.K.). Seedlings that emerged were thinned to seven per pot. Compost was kept moist by placing pots in propagator trays $(35.5 \times 21 \times 18 \mathrm{~cm})$, each containing a layer of water $\approx 1 \mathrm{~cm}$ deep. All plant material was raised in $1 \times 2.5 \times$ $1.3-\mathrm{m}$ controlled-environment $(\mathrm{CE})$ cabinets set at $18 / 15^{\circ} \mathrm{C}$ day/night temperatures and 16-h photoperiod, with a photosynthetic photon flux (measured at seedling height) ranging from 70 to $110 \mu \mathrm{mol}$ quanta $\mathrm{sec}^{-1} \mathrm{~m}^{-2}$. Seedlings were first inoculated 6 to 7 days after sowing, when cotyledons were fully expanded but true leaves were still developing (i.e., growth stage [GS] 1.0, as described by Sylvester-Bradley [21]). Inoculation of first and second true leaves usually was done 10 (GS 1.1) or 12 (GS 1.2) days after sowing. After inoculation, seedlings were returned to the incubation chamber under the same conditions, except for a transparent propagator lid that was placed over them to provide the high humidity required for successful infection.

Preparation of zoosporangia suspensions, inoculation, and disease assessment. Inoculum (zoosporangia suspensions) was prepared by shaking excised cotyledons supporting abundant sporulations in sterile distilled water (SDW) in a glass vial. Extraneous 
matter was removed from the resulting suspension by filtering through several layers of muslin. Before inoculation, zoosporangial suspensions were adjusted to the required concentration with a hemacytometer slide and appropriate dilution with SDW. In preliminary tests, zoosporangial germination was high $(>90 \%)$ and similar at each of the inoculum concentrations used in the experiments. Inoculation was done within 15 min of preparing zoosporangial suspensions.

Prior to inoculation, seedlings were sprayed with SDW to remove compost debris from their surfaces and left to dry for $30 \mathrm{~min}$. Inoculum was applied either by pipetting inoculum droplets onto cotyledons or by spraying seedlings to runoff with an atomizer. When droplets were used, a total of $10 \mu$ was applied per cotyledon as four $\approx 2.5$ - $\mu$ l droplets, two each on the adaxial surface of each half of a cotyledon. Droplets of this size were used, rather than larger ones, because they were never observed to run off the inoculated cotyledon. To test the local interaction between the IN and $\mathrm{CO}$ isolates, the isolates were applied either together as mixed inoculum or separately in succession as close as possible to the same site on the cotyledons. To test systemic interactions, various combinations of isolates were applied to opposite cotyledons. True leaves each received a total of $25 \mu \mathrm{l}$ of inoculum in similarly sized droplets pipetted onto their adaxial surfaces. Inoculum was agitated during application. Unless otherwise specified, inoculum concentrations of $1 \times 10^{5}$ (IN isolate) and $5 \times 10^{4}$ (CO isolate) zoosporangia per $\mathrm{ml}$ were used. In experiments that involved different treatments to opposite cotyledons, the position of each treatment was marked with a marker pen.

Disease reaction was assessed 7 days after inoculation, using a 0 to 9 scale (modified from Williams [26]) for both cotyledons and true leaves: $0=$ no symptoms or signs of $A$. candida infection; $1=$ pinpoint necrotic flecks at inoculation site, no sporulation; $2=$ larger necrotic flecks at inoculation site, no sporulation; $3=$ sparse sporulation, up to $5 \%$ of surface covered with pustules; $4=6$ to $10 \%$ of leaf area covered with pustules; $5=11$ to $20 \%$ leaf area covered with pustules; $6=21$ to $30 \%$ leaf area covered with pustules; $7=31$ to $50 \%$ leaf area covered with pustules; $8=51$ to $75 \%$ leaf area covered with pustules; and $9=>75 \%$ leaf area covered with pustules. At least seven seedlings were scored for each replication of a treatment combination.

All experiments involved three replications of each treatment, and propagator trays receiving different treatments were arranged randomly within CE cabinets. All experiments were repeated at least once. Mean disease severity was calculated for each tray, and the values were used in analysis of variance. $F$ tests were used to assess the significance of treatment main effects and interactions. Treatment means were differentiated using Fisher's least significant difference test (LSD). All analyses were done using the Genstat statistical package (Lawes Agricultural Trust, Hertfordshire, U.K.).

Local and systemic induction of resistance. In a preliminary experiment, batches of seedlings were either spray-inoculated with the IN isolate or sprayed with SDW; $4 \mathrm{~h}$ later all seedlings were spray-inoculated with the $\mathrm{CO}$ isolate. In a second experiment, seedlings were droplet-inoculated on one cotyledon (treatment A) with SDW or the IN isolate followed by the CO isolate and then inoculated on the other cotyledon (treatment $\mathrm{B}$ ) with either the $\mathrm{CO}$ or IN isolate. The possibility of systemic protection of true leaves was investigated in a third experiment: both cotyledons of a seedling were spray-inoculated with the IN isolate or with SDW as a control before the emergence of true leaves; 5 days later newly emerged true leaves were droplet-inoculated with the $\mathrm{CO}$ isolate.

Effect of IN inoculum concentration on $\mathrm{CO}$ infection. The $\mathrm{CO}$ isolate was droplet-inoculated either alone or in mixed suspension with increasing concentrations of the IN isolate $\left(0,5 \times 10^{3}\right.$, $1 \times 10^{4}, 5 \times 10^{4}, 1 \times 10^{5}$, and $2 \times 10^{5}$ zoosporangia per ml) on one cotyledon per seedling from different seedling batches; the opposite cotyledons of all seedlings received the $\mathrm{CO}$ isolate alone $\left(1 \times 10^{5}\right.$ zoosporangia per $\left.\mathrm{ml}\right)$. Mixed suspensions were prepared in such a way that the inoculum concentration of the CO isolate remained constant.

Importance of timing and sequence of IN and $\mathrm{CO}$ inoculations. Both cotyledons of each seedling in a batch received droplet inoculations with both the IN and CO isolates, but the inoculations were staggered so there were different time intervals between the applications of each isolate. Both IN followed by $\mathrm{CO}$ and $\mathrm{CO}$ followed by IN combinations were tested in four experiments. (i) Intervals of $4 \mathrm{~h}$ between initial application of the $\mathrm{CO}$ or IN isolate or $\mathrm{H}_{2} \mathrm{O}$ followed by application of the $\mathrm{CO}$ or $\mathrm{IN}$ isolate or $\mathrm{H}_{2} \mathrm{O}$ compared with simultaneous application of the $\mathrm{CO}$ and IN isolates. (ii) Cotyledons were first inoculated with $\mathrm{H}_{2} \mathrm{O}$ or the IN isolate and subsequently inoculated with the $\mathrm{CO}$ isolate $0,1,3,5$, or 7 days later. In similar experiments, true leaves 1 and 2 were inoculated with the $\mathrm{CO}$ isolate 5 or 7 days and 7 days, respectively, after initial inoculation of cotyledons with $\mathrm{H}_{2} \mathrm{O}$ or the IN isolate. Results for cotyledons and true leaves 1 and 2 were analyzed separately. (iii) Cotyledon 1 was inoculated initially with $\mathrm{H}_{2} \mathrm{O}$ or the IN isolate, and both cotyledons were inoculated with the $\mathrm{CO}$ isolate after 0,1 , or 3 days. (iv) Cotyledons were initially inoculated with the $\mathrm{CO}$ isolate and then with the IN isolate after $0,1,3$, or 5 days. Cotyledons of control seedlings were inoculated a second time with $\mathrm{H}_{2} \mathrm{O}$. In all cases, disease reaction was scored 7 days after last inoculation.

Microscope observation of zoospore distribution. A subsample of four cotyledons was collected $4 \mathrm{~h}$ after droplet-inoculation with the IN isolate alone. Cotyledons were washed thoroughly three times in SDW in a glass vial and mounted in a $0.1 \%$ aqueous solution of Calcofluor on microscope slides. The location of zoospores in relation to stomata was investigated with a fluorescence microscope (Leitz, Wetzlar, Germany) fitted with a 390to $440-\mathrm{nm}$ exciter filter and a $475-\mathrm{nm}$ barrier filter (22). The extent of zoospore lodging over stomata was estimated by counting the number of zoospores over, or away from, 100 stomata under the site of the inoculum droplet on each replicate cotyledon.

Phenylalanine ammonia lyase and total soluble peroxidase assays. Subsamples of cotyledons were collected at the time of dropletinoculation with the IN or CO isolate and 1, 3, and 5 days after inoculation. SDW-treated cotyledons were sampled at the same times for the control. For each treatment combination, a 2-g sample was homogenized in $20 \mathrm{ml}$ of homogenization buffer $(0.1 \mathrm{M}$ potassium phosphate, $50 \mathrm{mM}$ sodium metabisulphite, $1 \mathrm{mM}$ phenylmethylsulphonyl fluoride, and $250 \mathrm{mM}$ sucrose, $\mathrm{pH}$ 7.0). The homogenate was mixed with $2.5 \mathrm{~g}$ of polyvinylpolypyrrolidone (standard grade, Sigma Chemical Co., St. Louis) and $1.25 \mathrm{~g}$ of Amberlite XAD-4 (standard grade, Sigma) ion-exchange resin for $2 \mathrm{~min}$, filtered through two layers of muslin cloth, and centrifuged at $100,000 \times g$ at $4^{\circ} \mathrm{C}$ for $30 \mathrm{~min}$. A $1-\mathrm{ml}$ aliquot of supernatant was purified further and desalted by centrifugation through a 2-ml Sepharose $\mathrm{G} 25$ mini-column (3,000 rpm for $5 \mathrm{~min}$ at $4^{\circ} \mathrm{C}$ ). The $\mathrm{G} 25$ column removes excess salt and simple organic acids that might interfere with both the phenylalanine ammonia lyase (PAL) and total soluble peroxidase (POX) assays. PAL and POX activities were assayed by procedures modified from those reported by Strack and Mock (20). For PAL, $50 \mu \mathrm{l}$ of supernatant was mixed with $950 \mu \mathrm{l}$ of substrate buffer $(10 \mathrm{mM}$ L-phenylalanine in $100 \mathrm{mM}$ potassium borate, $\mathrm{pH} 8.8$ ), and the formation of trans-cinnamic acid was measured at $290 \mathrm{~nm}$ after $2 \mathrm{~h}$ of incubation at $30^{\circ} \mathrm{C}$. PAL activity was expressed as nanomoles trans-cinnamic acid per gram fresh weight per hour. For POX, $50 \mu \mathrm{l}$ of supernatant was mixed with $950 \mu \mathrm{l}$ of substrate buffer $(4.5 \mathrm{mM}$ guaiacol [50 $\mu \mathrm{l}], 2.2 \mathrm{mM}$ hydrogen peroxide [25 $\mu$ lof $30 \%$ stock], and $200 \mathrm{mM}$ potassium phosphate, $\mathrm{pH}$ 5.8). The formation of tetraguaiacol was recorded spectrophotometrically at $30^{\circ} \mathrm{C}$ for $2 \mathrm{~min}$, and a rate was determined. POX activity was expressed as nanomoles tetraguaiacol formed per gram fresh weight per hour. Six assays for both PAL and POX were performed for each sample. 


\section{RESULTS}

Local and systemic induction of resistance. A preliminary experiment indicated that pretreatment by spraying with the IN isolate protected seedlings from the $\mathrm{CO}$ isolate (disease reaction $=$ 3.92 versus 7.06 for the control; standard error of difference between means $=0.326 ; F$ significant at $P=0.0006$ ). The relative virulence of each isolate on $B$. juncea accession PPBJ- 1 and the local and systemic induction of resistance to the $\mathrm{CO}$ isolate by the IN isolate are shown in Figure 1. Within the time (6 to 7 days) it took the $\mathrm{CO}$ isolate to produce abundant white pustules on the lower surfaces of cotyledons (Fig. 1A), the IN isolate produced few symptoms. Occasionally, IN-inoculated cotyledons became slightly curled, but they had a disease reaction of only 0 or 1 . Coinoculation with the IN and $\mathrm{CO}$ isolates simultaneously on one cotyledon decreased disease reaction on the cotyledon, as well as on the opposite cotyledon inoculated with the CO isolate alone (Fig. 1B).
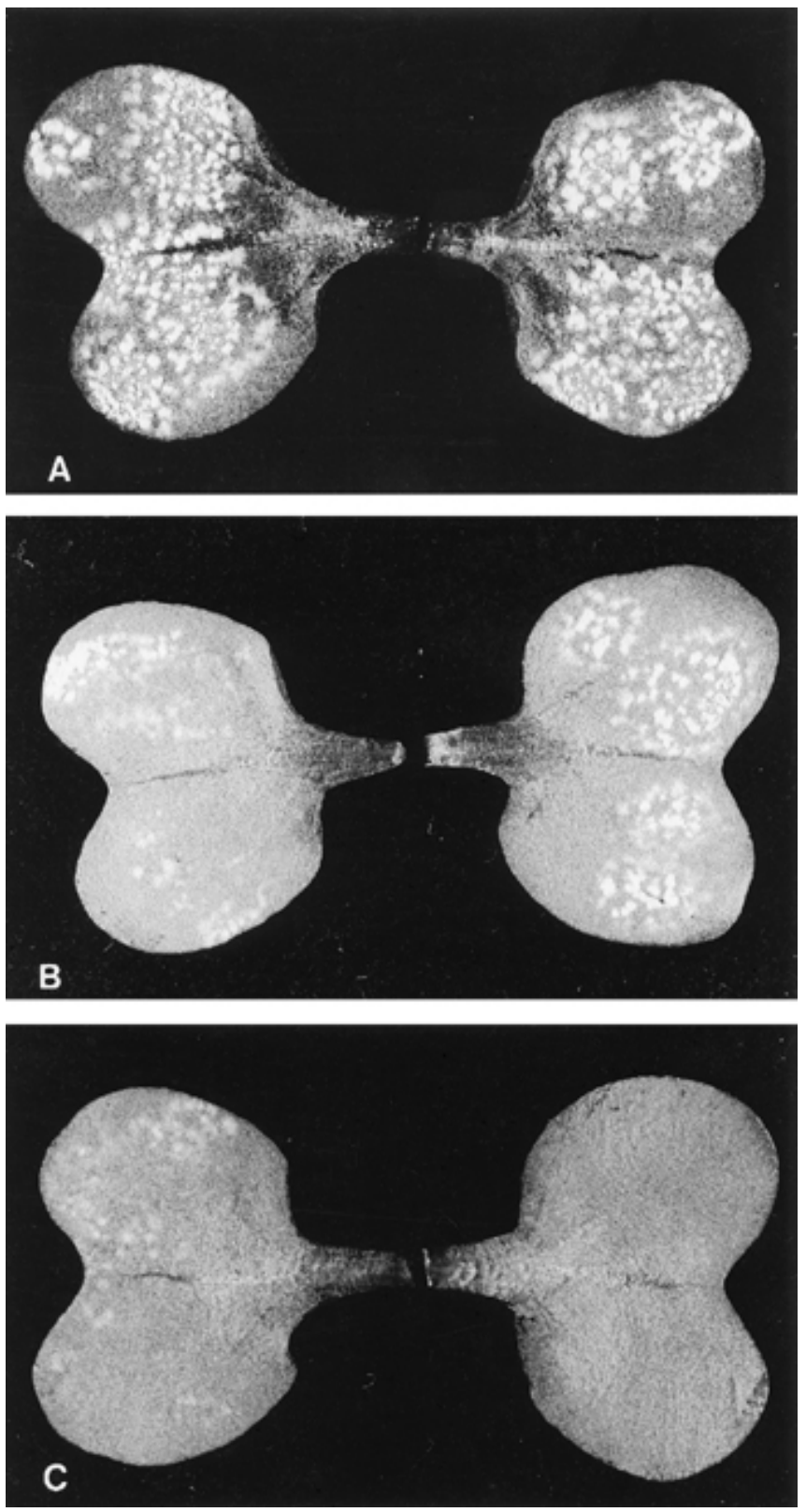

Fig. 1. Symptom development on cotyledons of Brassica juncea accession PPBJ-1 7 days after inoculation with incompatible (IN) and compatible (CO) isolates of Albugo candida. A, CO isolate inoculated on both cotyledons; B, $\mathrm{CO}$ and IN isolates inoculated together on left cotyledon; $\mathrm{CO}$ isolate applied alone to right cotyledon; $\mathbf{C}, \mathrm{CO}$ and $\mathrm{IN}$ isolates inoculated together on left cotyledon; IN isolate applied alone to right cotyledon

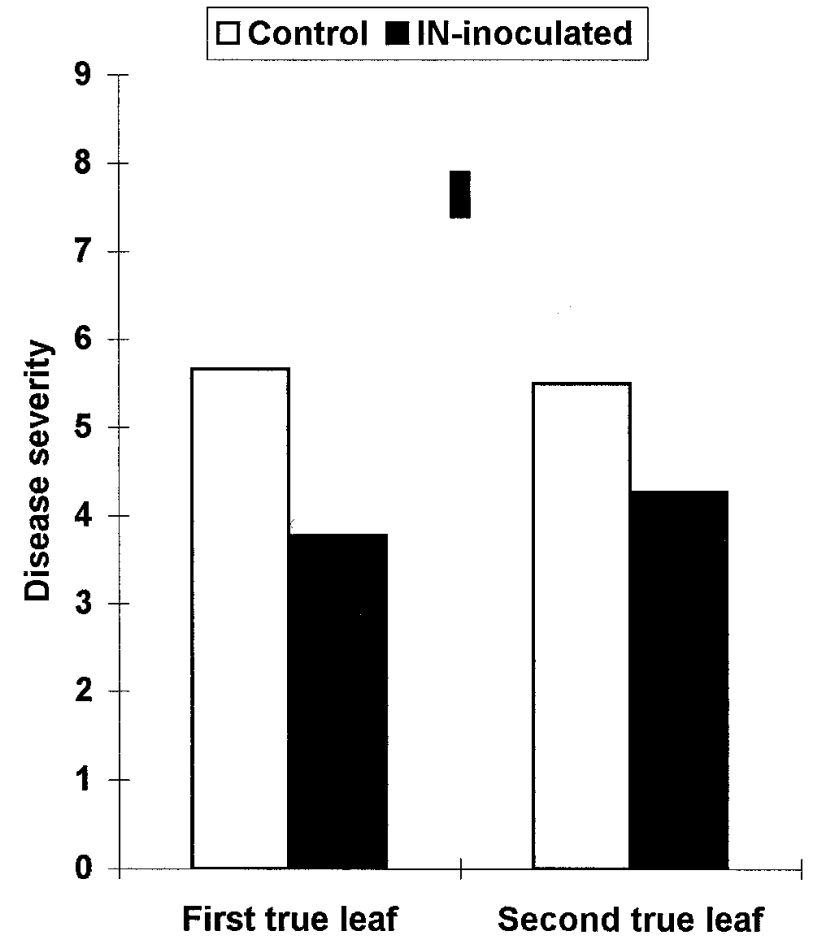

Fig. 2. White rust severity on first and second true leaves of Brassica juncea when the compatible Albugo candida isolate was applied 5 days after both cotyledons had been sprayed either with sterile distilled water (control) or inoculated with the incompatible (IN) isolate. Bar represents $\operatorname{LSD}_{(P=0.05, \mathrm{df}=8)}$.

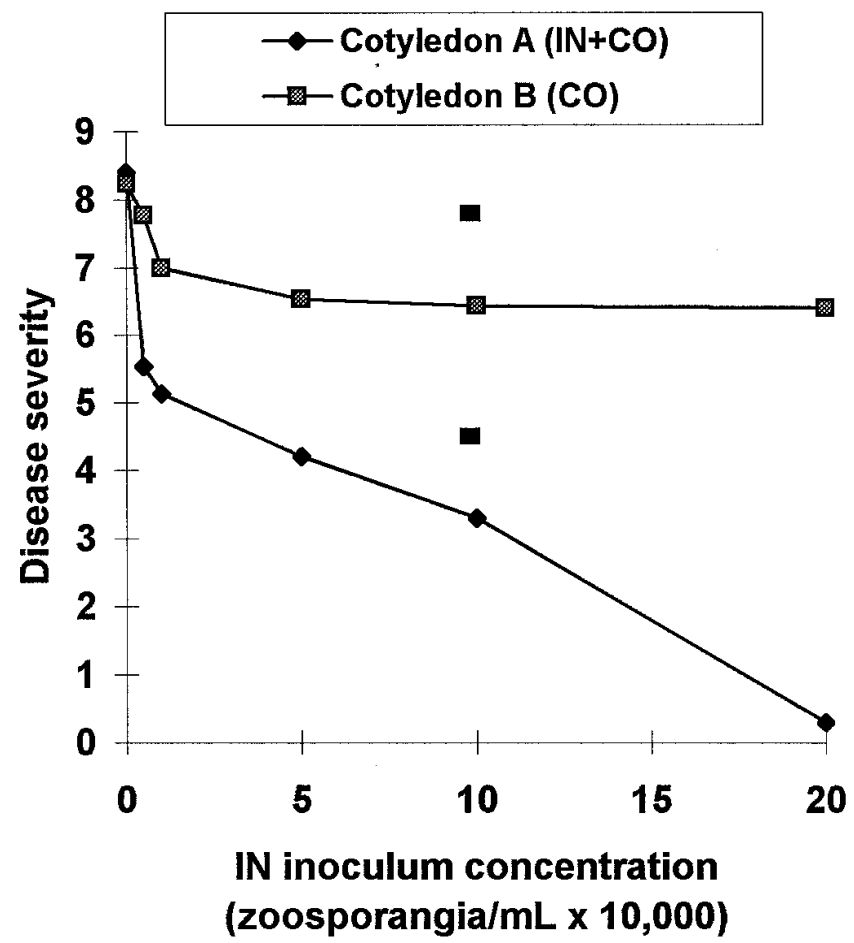

Fig. 3. Effect of incompatible (IN) isolate (Albugo candida) inoculum concentration on disease severity on Brassica juncea after inoculation with the compatible (CO) isolate. On each seedling, cotyledon A was inoculated with both the IN and CO isolates; at the same time, cotyledon B was inoculated with the $\mathrm{CO}$ isolate alone. The top bar represents $\operatorname{LSD}_{(P=0.05, \mathrm{df}=24)}$ for comparisons at different inoculum concentrations. The bar between the lines represents $\operatorname{LSD}_{(P=0.05, \mathrm{df}=12)}$ for comparisons of disease reaction on the two cotyledons at a particular IN inoculum concentration. 
However, systemic protection (on the opposite cotyledon) was not as great as local protection. If the opposite cotyledon was inoculated instead with the IN isolate after the first simultaneous IN and $\mathrm{CO}$ inoculation, there was no change in disease reaction on the cotyledon (Fig. 1C). There was no evidence that coinoculation with the two isolates encouraged infection by the IN isolate.

Inoculating cotyledons with the IN isolate also protected subsequently emerging true leaves against the $\mathrm{CO}$ isolate $(P<0.001)$. The first and second true leaves were protected to a similar extent ( $P=0.064$ for the $F$ test for leaf-isolate interaction; Fig. 2).

Effect of IN inoculum concentration on $\mathrm{CO}$ infection. The degrees of both local and systemic protection of cotyledons against the CO isolate increased as the concentration of the IN isolate used in mixed inoculations increased $(P<0.001)$. Local protection was greater than systemic protection $(P<0.001$; Fig. 3$)$. The pattern of protection differed between local and systemic $(P<0.001)$. Systemic protection was greatest at $\approx 5 \times 10^{4}$ zoosporangia per $\mathrm{ml}$ (Fig. 3, cotyledon B), whereas local protection continued to increase up to the highest concentration of IN isolate applied $\left(2 \times 10^{5}\right.$ zoosporangia per ml; Fig. 3, cotyledon A).

Importance of timing and sequence of IN and $\mathrm{CO}$ inoculations. The local protection given by inoculation with the IN isolate increased when there was a delay of as little as $4 \mathrm{~h}$ before

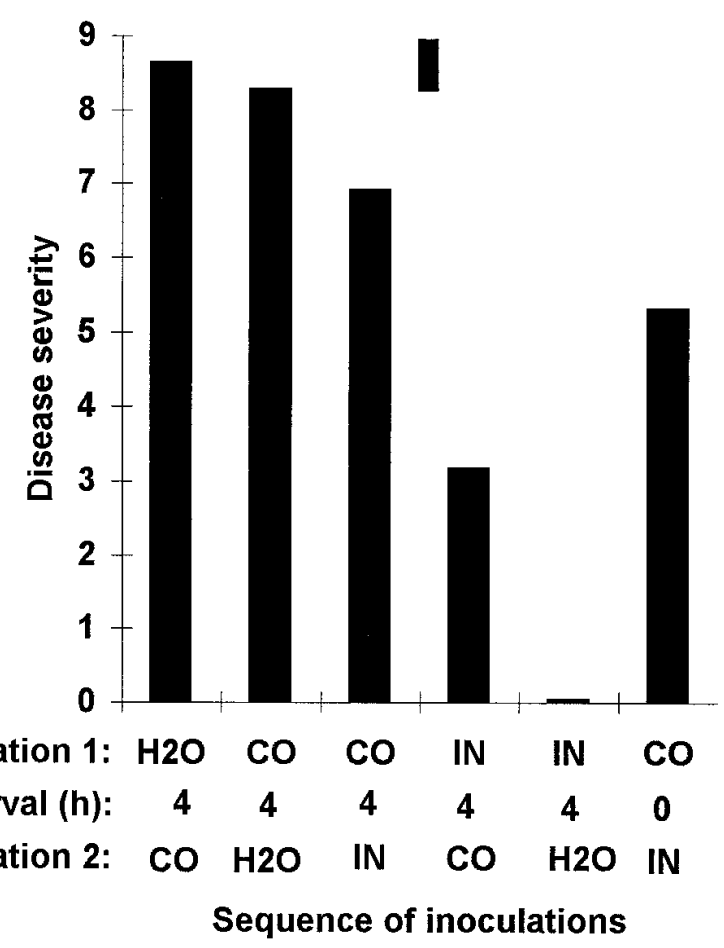

Fig. 4. Effect of sequence of inoculation of Brassica juncea cotyledons with the incompatible (IN) and compatible (CO) isolates of Albugo candida on local protection against the $\mathrm{CO}$ isolate. The bar represents $\left.\left.\operatorname{LSD}_{(P=0.05} \mathrm{df}=10\right)\right)$ for all means, except that of the $\mathrm{IN} / 4 \mathrm{~h} / \mathrm{H}_{2} \mathrm{O}$ treatment, which was excluded from the analysis. challenge inoculation with the CO isolate (Fig. 4). Disease severity was reduced compared with cotyledons inoculated with the $\mathrm{CO}$ isolate after $4 \mathrm{~h}$ of inoculation with $\mathrm{H}_{2} \mathrm{O}$, whether inoculation with the IN isolate took place before or after inoculation with the $\mathrm{CO}$ isolate $(P<0.001)$. However, inoculation with the IN isolate $4 \mathrm{~h}$ before the $\mathrm{CO}$ isolate gave the greatest protection; protection was weaker with simultaneous inoculation with the $\mathrm{CO}$ and IN isolates and weakest with inoculation with the IN isolate $4 \mathrm{~h}$ after inoculation with the $\mathrm{CO}$ isolate $(P<0.001$; Fig. 4$)$.

There was clear evidence of both local protection of cotyledons and systemic protection of true leaves induced by initial inoculation of cotyledons with the IN isolate $(P<0.001$; Fig. 5). Local protection of cotyledons and systemic protection of leaves increased as the delay until inoculation with the $\mathrm{CO}$ isolate increased $(P<$ 0.001 ), but there appeared to be a decline in the systemic protection of true leaves, relative to control, when the interval was extended from 5 to 7 days. However, the difference in disease severity between the control and IN inoculation decreased as the delay until inoculation with the $\mathrm{CO}$ isolate increased $(P=0.001)$.

There also was evidence of both local and systemic protection of cotyledons induced by the IN inoculation $(P<0.001)$, but sys-

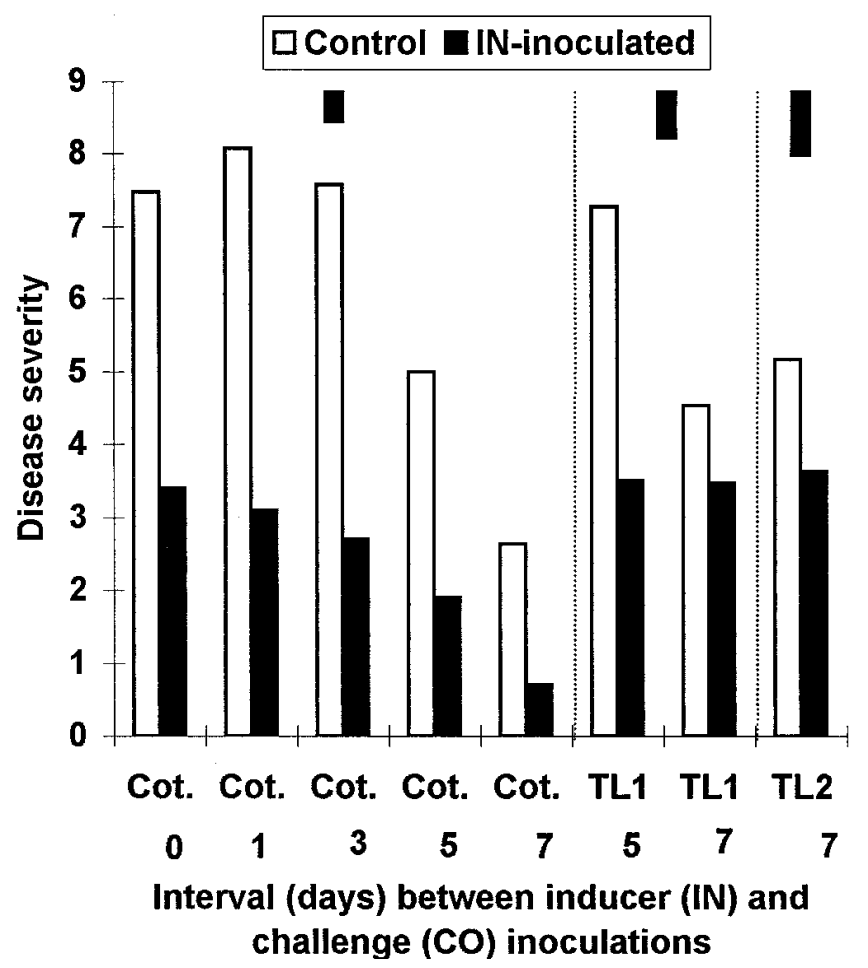

Fig. 5. Duration of local protection of Brassica juncea cotyledons (Cot.) and systemic protection of true leaves (TL) against challenge inoculation with the Albugo candida compatible (CO) isolate after inducing inoculation of cotyledons with the incompatible (IN) isolate. Both cotyledons of a seedling were treated identically. Bars represent $\operatorname{LSD}_{(P=0.05)}$ of disease severity on cotyledons $(\mathrm{df}=20)$, first true leaves $(\mathrm{df}=8)$, and second true leaves $(\mathrm{df}=4)$.

TABLE 1. Importance of the interval between inoculation with incompatible (IN) and compatible (CO) isolates of Albugo candida in relation to induced local (cotyledon 1) and systemic (cotyledon 2) protection against white rust in Brassica juncea ${ }^{\mathrm{a}}$

\begin{tabular}{|c|c|c|c|c|c|c|c|}
\hline Interval (days) & \multicolumn{3}{|c|}{ Cotyledon $1^{\mathrm{a}}$} & \multicolumn{3}{|c|}{ Cotyledon $2^{\mathrm{a}}$} & $\begin{array}{c}\text { Cotyledon } 1- \\
\text { cotyledon } 2\end{array}$ \\
\hline 0 & $\mathrm{H}_{2} \mathrm{O}$ & $\mathrm{CO}$ & 8.13 & - & $\mathrm{CO}$ & 8.00 & +0.13 \\
\hline \multirow[t]{2}{*}{1} & $\mathrm{H}_{2} \mathrm{O}$ & $\mathrm{CO}$ & 7.80 & - & $\mathrm{CO}$ & 7.73 & +0.07 \\
\hline & IN & $\mathrm{CO}$ & 5.00 & - & $\mathrm{CO}$ & 6.67 & -1.67 \\
\hline \multirow[t]{2}{*}{3} & $\mathrm{H}_{2} \mathrm{O}$ & $\mathrm{CO}$ & 6.47 & - & $\mathrm{CO}$ & 6.33 & +0.14 \\
\hline & IN & $\mathrm{CO}$ & 3.57 & - & $\mathrm{CO}$ & 4.83 & -1.26 \\
\hline
\end{tabular}

${ }^{\mathrm{a}} \operatorname{LSD}_{(P=0.05, \mathrm{df}=24)}=0.44$ 
temic protection was lower $(P<0.001$; Table 1$)$. Disease severity in general decreased as the delay until inoculation with the $\mathrm{CO}$ isolate increased, but there was no evidence of any change in this pattern as the length of the delay increased $(P>0.35$ for all interactions with delayed inoculation with the $\mathrm{CO}$ isolate).

In another experiment, disease severity was reduced by $\approx 50 \%$ when cotyledons were simultaneously inoculated with the $\mathrm{CO}$ and IN isolates compared with those inoculated simultaneously with the $\mathrm{CO}$ isolate and $\mathrm{H}_{2} \mathrm{O}$, but the difference became minimal as the delay until inoculation with the IN isolate increased to 5 days (Fig. 6).

Zoospore distribution on inoculated cotyledon surfaces. At the zoosporangia concentration used for the IN isolate $\left(1 \times 10^{5}\right.$ zoosporangia per $\mathrm{ml})$, almost all $(>97 \%)$ stomata under the inoculum droplet were occupied by encysted zoospores.

PAL and POX activities in inoculated tissues. PAL and POX activities declined in uninoculated cotyledons during the course of the assays, but inoculated cotyledons showed net increases in the activities of both enzymes, and the difference from the control increased with time after inoculation $(P<0.001)$. The activities of both enzymes were consistently higher in IN- than in CO-inoculated cotyledons; the rate of increase in POX activity was similar for the two isolates, but the rate of increase in PAL activity was greater with the IN isolate (Fig. 7A and B). Cotyledons and true leaves that had been coinoculated with IN and CO isolates simultaneously were not assessed.

\section{DISCUSSION}

Attempted infection of cotyledons by an IN isolate of $A$. candida provided various degrees of protection of $B$. juncea seedlings, both local and systemic, against an $\mathrm{CO}$ isolate. The degree of protection depended on the zoosporangia concentration of IN isolate applied, sequence of inoculations, and interval between inoculations with the IN and $\mathrm{CO}$ isolates. However, the $\mathrm{CO}$ isolate only produced symptoms comparable with an IN reaction when a very high concentration of inducing (IN) inoculum was used. The protective

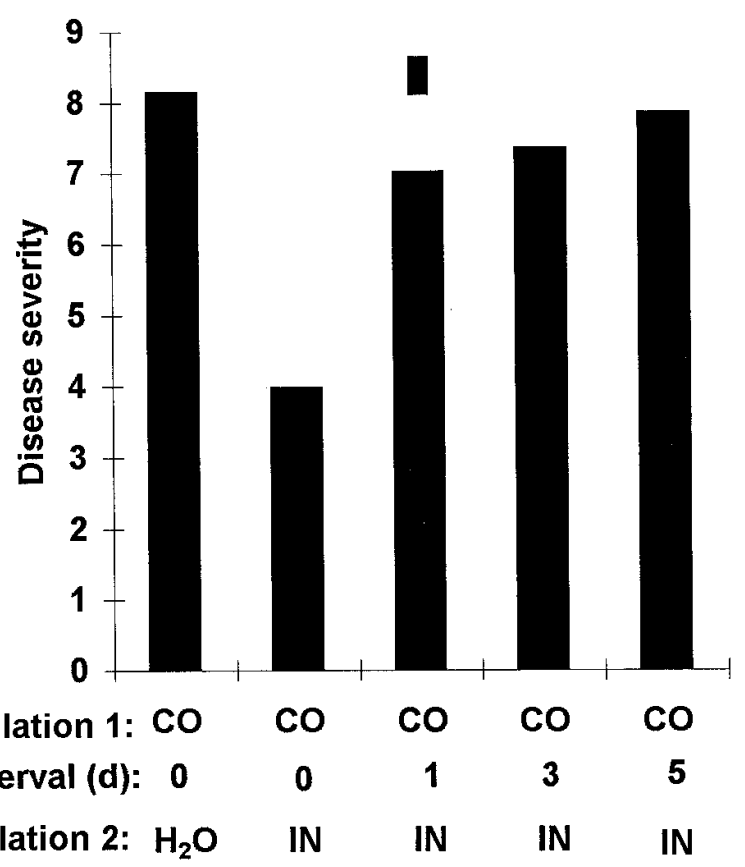

\section{Sequence of inoculation}

Fig. 6. Effect of inoculation with the incompatible (IN) Albugo candida isolate on disease severity on Brassica juncea when applied at different intervals after inoculation with the compatible (CO) isolate. Both cotyledons of a seedling were treated identically. The bar represents the $\operatorname{LSD}_{(P=0.05, \mathrm{df}=10)}$. effect was also greatest locally and appeared to decline in tissues remote from the point of IN isolate inoculation.

This interaction probably was not due to direct antagonism between zoospores of the two isolates. A more likely explanation is a combination of two effects: competition between the isolates for infection sites and inhibition of the $\mathrm{CO}$ isolate by host resistance responses induced by the IN isolate.
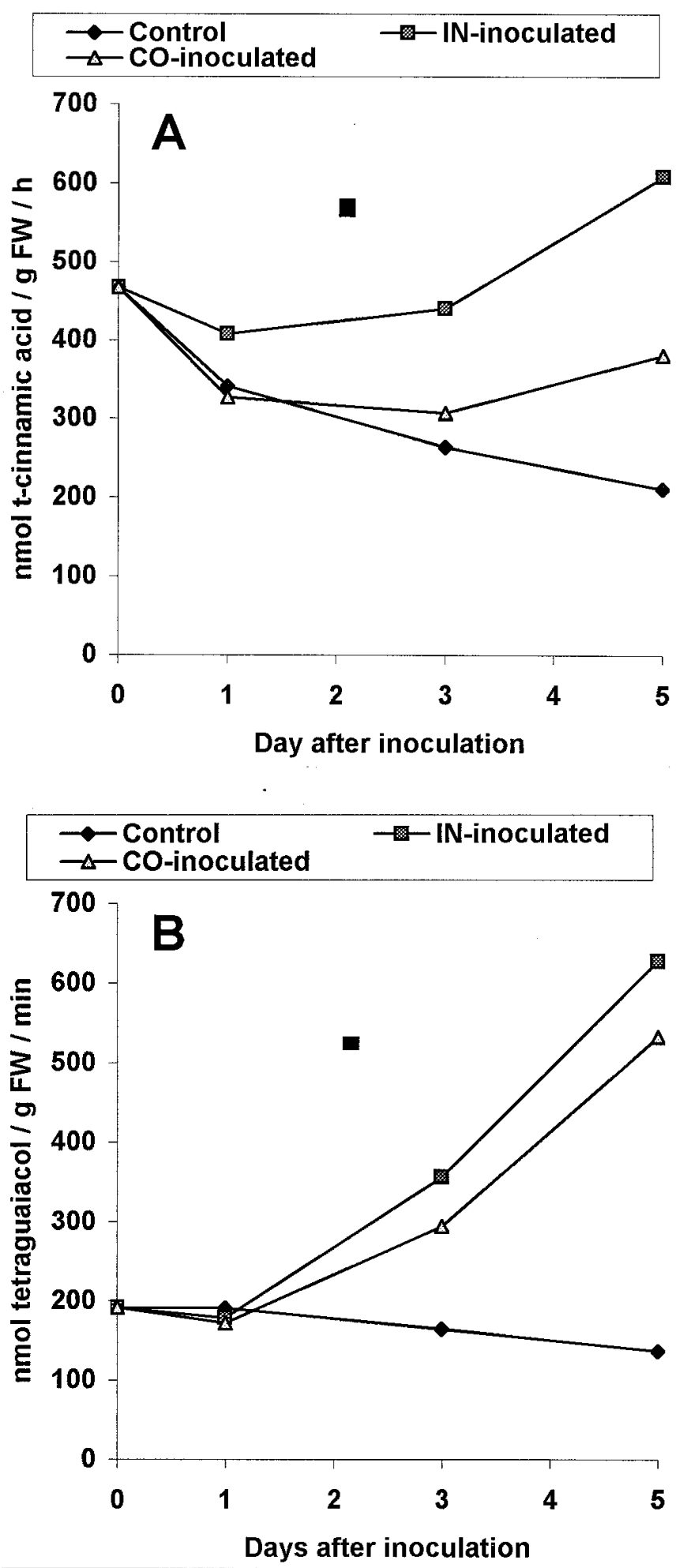

Fig. 7. A, Phenylalanine ammonia lyase (PAL) and $\mathbf{B}$, total soluble peroxidase (POX) activities in Brassica juncea cotyledons after inoculation of seedlings (both cotyledons simultaneously) with either the compatible (CO) or incompatible (IN) Albugo candida isolates. The bars represent the $\operatorname{LSD}_{(P=0.05)}$ when $\mathrm{df}=44$ and 22 for $\mathbf{A}$ and $\mathbf{B}$, respectively. 
Competition for infection sites is likely to have contributed to local protection conferred by the IN isolate, at least in treatments involving IN isolate inoculation before the $\mathrm{CO}$ isolate. A. candida zoospores must lodge over a stoma to initiate infection, and they must do so in an orientation that allows the emerging germ tube to grow directly into the stomatal chamber. Zoospores of the two isolates used in our study germinate from zoosporangia, encyst, and send germ tubes into stomatal chambers at a similar rate. More than one zoospore can lodge over a stoma, but once the stoma is occupied, the presence of one zoospore appears to reduce the likelihood that subsequent zoospores will lodge and penetrate successfully (U. S. Singh, K. J. Doughty, N. I. Nashaat, G. Ross, and S. J. Kolte, unpublished data). When the IN and $\mathrm{CO}$ isolates were inoculated together, the IN isolate is likely to have prevented a significant proportion of the $\mathrm{CO}$ isolate zoospores from penetrating successfully, particularly when a high IN zoosporangia concentration was applied. The fact that local protection was greater when the IN isolate inoculum preceded the $\mathrm{CO}$ isolate inoculum by as little as $4 \mathrm{~h}$ and less when the IN isolate followed the $\mathrm{CO}$ isolate than when the two isolates were inoculated together is consistent with this hypothesis. Four hours between successive inoculations with the IN and CO isolates is long enough to allow zoospores of the IN isolate to deny the $\mathrm{CO}$ isolate a proportion of stomatal infection sites by blocking them. Also, increasing the delay between inducing (IN) and challenge (CO) inoculations did not greatly increase the extent of local protection, and there was no significant "curative" effect when the IN isolate was applied more than 1 day after the $\mathrm{CO}$ isolate.

Systemic protection against the $\mathrm{CO}$ isolate conferred by the IN isolate was host-mediated. Local protection conferred by the IN isolate probably also was host-mediated, at least in part. For example, treatments that involved inoculating a cotyledon with the $\mathrm{CO}$ isolate and then the IN isolate (precluding the possibility of denial of infection sites by the IN isolate) also decreased the extent of local symptom development. However, local symptom development was much less when the isolates were inoculated at the same time or when the IN isolate was inoculated first. It is difficult to estimate the relative contributions of competition for infection sites and induced resistance to the local interaction between the two isolates.

Induced (IN) responses were likely to be relatively rapid ones, coinciding perhaps with inhibition of haustorial development in the mesophyll, which appears to be the first indication of an IN interaction $(11,23)$. The IN isolate probably was recognized by the host relatively early, and its attempt to infect may have led to the release of elicitors that condition the expression of plant resistance genes-the products of which may inhibit infection by the $\mathrm{CO}$ isolate. This is consistent with the earlier and greater increase in PAL activity and the slightly greater increase in POX activity after inoculation with the IN isolate than with the CO isolate. Visible symptoms of hypersensitive reactions to fungal infection commonly occur between 3 and 5 days after inoculation. However, there are clearly other defense-related events that occur much earlier in resistant and hypersensitive plants, including insolubilization of cell wall proteins and induction of various enzymes and crucifer indole phytoalexins $(15,19)$. Both PAL and POX are associated with induced resistance in various species and have various functions, including control of key stages in biosynthesis of lignin precursors (PAL), strengthening of cell walls against pathogen invasion (PAL and POX), and biosynthesis of indolylglucosinolates and indole phytoalexins (POX) $(4,7,25)$. Dahiya and Woods (5) reported that infection by $A$. candida induced production of fungitoxic phytoalexins in rapeseed (species unspecified). It is possible that increased PAL and POX activities may contribute to protection of $B$. juncea against the $\mathrm{CO}$ isolate conferred by the IN isolate. However, Brassica species also are capable of other biochemical responses to infection that are not measured here, including production of pathogenesis-related proteins and glucosinolates $(6,8,19)$. Further studies need to be completed before the precise biochemical mechanism(s) of the IN isolate-induced resistance can be identified.

The greater local and systemic protection resulting from increased inducing (IN) inoculum corresponds with other studies (14). However, when increasing inoculum concentrations of the IN isolate were applied to one cotyledon, there appeared to be a limit to the extent of protection of opposite cotyledons inoculated with the $\mathrm{CO}$ isolate, such that the response (and presumably the signaling mechanism that invokes it) were greatest at relatively low inducing zoosporangia concentrations.

The pattern of interactions between the $\mathrm{CO}$ and IN isolates of $A$. candida on $B$. juncea corresponded, to some extent, to those found between highly virulent (HV) and weakly virulent (WV) isolates of Leptosphaeria maculans on B. napus (12). However, in contrast to $A$. candida, the optimum interval between WV and HV L. maculans inoculations was $24 \mathrm{~h}$, and it was possible to achieve a curative effect up to $48 \mathrm{~h}$ after $\mathrm{HV}$ inoculation.

Our controlled-environment studies suggest there are likely interactions in the field among pathotypes of $A$. candida expressing different host specificities. In similar work, Voorrips (24) concluded that induction of host resistance was a better explanation for interactions between Plasmodiophora brassicae isolates differing in virulence on $B$. oleracea than competition for infection sites. In the $A$. candida-B. juncea system, both competition and induced resistance may be involved, although the relative importance of each in nature is determined by the amount of avirulent inoculum present. In western Canada, where both B. rapa and B. juncea are grown, there is a predominance of isolates that infect only $B$. rapa (13), and these might be expected to interfere with infection by isolates specific to $B$. juncea. The scale and significance of these interactions in crops depends not only on the virulence composition of the local pathogen population, but also on the relative timing and proximity of cultivation of respective hosts and on the likelihood that $A$. candida races differing in virulence coexist at infection sites, as is the case for the L. maculans-B. napus (12) and other (1) systems. In our study, the optimum local protection that occurred after preinoculation with the IN isolate alone approached a response comparable to an IN interaction when a high zoosporangia concentration $\left(2 \times 10^{5} \mathrm{ml}^{-1}\right)$ was used. The importance of the timing and sequence of IN and $\mathrm{CO}$ inoculations to the induction of resistance to $A$. candida in B. juncea suggests that major effects in the field are less likely than for L. maculans, for which induced resistance appears to be more flexible (12).

Based on the results presented in our paper, biological control of $A$. candida based on intervention with IN isolates appears difficult to achieve. The use of antagonistic or resistance-inducing bacterial inoculants for control may be a more promising approach (9). However, the $A$. candida-B. juncea interactions described here may provide a useful model for identifying the systemic biochemical responses that determine incompatibility.

\section{ACKNOWLEDGMENTS}

We thank the U.K. Department for International Development for financial support, S. Welham for advice on presentation of statistical analyses, and A. Heran for technical assistance.

\section{LITERATURE CITED}

1. Adachi, Y., and Tsuge, T. 1994. Coinfection by different isolates of Alternaria alternata in single black point lesions of Japanese pear leaves. Phytopathology 84:447-451.

2. Awasthi, R. P., Nashaat, N. I., Heran, A., Kolte, S. J., and Singh, U. S. 1997. The effect of Albugo candida on the resistance to Peronospora parasitica and vice versa in rapeseed-mustard. Page 49 in: Abstr. ISHS Symp. Brassicas: 10th Crucifer Genet. Workshop. ENSAR-INRA, Rennes, France.

3. Bisht, I. S., Agrawal, R. C., and Singh, R. 1994. White rust (Albugo candida) severity in mustard (Brassica juncea) and its effect on seed yields. Plant Var. \& Seeds 7:85-89.

4. Bowles, D. J. 1990. Defense-related proteins in higher plants. Annu. Rev. 
Biochem. 59:873-907.

5. Dahiya, J. S., and Woods, D. L. 1987. Phytoalexin accumulation in rapeseed leaves challenged with white rust (Albugo candida). (Abstr.) Can. J. Plant Pathol. 9:276.

6. Dixelius, C. 1994. Presence of pathogenesis-related proteins 2, Q and S in stressed Brassica napus and Brassica nigra plantlets. Physiol. Mol. Plant Pathol. 44:1-8.

7. Dixon, R. A., and Paiva, N. L. 1995. Stress-induced phenylpropanoid metabolism. Plant Cell 7:1085-1097.

8. Doughty, K. J., Porter, A. J. R., Morton, A. M., Kiddle, G., Bock, C. H., and Wallsgrove, R. M. 1991. Variation in the glucosinolate content of oilseed rape (Brassica napus L.) leaves. II. Response to infection by Alternaria brassicae (Berk.) Sacc. Ann. Appl. Biol. 118:469-477.

9. Goyal, B. K., Verma, P. R., and Reddy, M. S. 1995. Suppression of white rust on mustard by foliar and seed application of microbial inoculants. (Abstr.) Can. J. Plant Pathol. 17:357.

10. Hammerschmidt, R., and Kuć, J., eds. 1995. Induced Resistance to Disease in Plants. Developments in Plant Pathology 4. Kluwer Academic Publishers, Dordrecht, Netherlands.

11. Liu, Q., Rimmer, S. R., and Scarth, R. 1989. Histopathology of compatibility and incompatibility between oilseed rape and Albugo candida. Plant Pathol. 38:176-182.

12. Mahuku, G. S., Hall, R., and Goodwin, P. H. 1996. Co-infection and induction of systemic acquired resistance by weakly and highly virulent isolates of Leptosphaeria maculans in oilseed rape. Physiol. Mol. Plant Pathol. 49:61-72.

13. Mathur, S., Wu, C. R., and Rimmer, S. R. 1995. Pathogenic variation among Albugo candida isolates from Western Canada. (Abstr.) Phytopathology 85:1175.

14. O’Neill, N. R., Elgin, J. H., and Baker, C. J. 1989. Characterization of induced resistance to anthracnose in alfalfa by races, isolates, and species of Colletotrichum. Phytopathology 79:750-756.

15. Otte, O., and Barz, W. 1996. The elicitor-induced oxidative burst in cultured chickpea cells drives the rapid insolubilization of two cell wall structural proteins. Planta 200:238-246.

16. Petrie, G. A. 1988. Races of Albugo candida (white rust and staghead) on cultivated Cruciferae in Saskatchewan. Can. J. Plant Pathol. 10:142-150.

17. Pidskalny, R. S., and Rimmer, S. R. 1985. Virulence of Albugo candida from turnip rape (Brassica campestris) and mustard (B. juncea) on various crucifers. Can. J. Plant Pathol.7:283-286.

18. Sequira, L. 1983. Mechanisms of induced resistance in plants. Annu. Rev. Microbiol. 37:51-79.

19. Shewry, P. R., and Lucas, J. A. 1997. Plant proteins that confer resistance to pests and pathogens. Adv. Bot Res. 26:135-192.

20. Strack, D., and Mock, H. P. 1993. Enzymes of secondary plant metabolism. Methods Plant Biochem. 9:45-98.

21. Sylvester-Bradley, R. 1985. Revision of a code for stages of development in oilseed rape (Brassica napus L.). Aspects Appl. Biol. 10:395-400.

22. Trese, A. T., and Loschke, D. C. 1990. High contrast resolution of the mycelia of pathogenic fungi in corn tissue after staining with Calcofluor and destaining with cellulase. Phytopathology 80:196-200.

23. Verma, P. R., Harding, H., Petrie, G. A., and Williams, P. H. 1975. Infection and temporal development of Albugo candida in cotyledons of four Brassica species. Can. J. Bot.53:1016-1020.

24. Voorrips, R. E. 1996. Production, characterization and interaction of singlespore isolates of Plasmodiophora brassicae. Eur. J. Plant Pathol. 102:377383.

25. Wallsgrove, R. M., Doughty, K. J., and Bennett, R. N. 1990. Glucosinolates. Pages 523-562 in: Plant Amino Acids, B. J. Singh, ed. Marcel Dekker Inc., New York.

26. Williams, P. H. 1985. Crucifer Genetics Cooperative (CrGC) Resource Book. Department of Plant Pathology, University of Wisconsin, Madison. 